\title{
O Problema do Brigadista com Vértices Resistentes
}

\author{
Luis F. L. Sales ${ }^{1}$, Raimundo A. Neto ${ }^{1}$, Glauber F. Cintra ${ }^{1}$ \\ ${ }^{1}$ Instituto Federal de Educação, Ciência e Tecnologia do Ceará (IFCE)
}

luisfilipels@hotmail.com, raimundonetoabclgmail.com, glauberfcintralgmail.com

\begin{abstract}
This paper presents a new variant of the Firefighter Problem, namely, the Firefighter Problem with Resistant Vertices, that distinguishes itself through the existence of an integer fire resistance associated with each vertex. We present five heuristics to the problem and the results obtained in computational tests.
\end{abstract}

Resumo. Este artigo apresenta uma nova variante do Problema do Brigadista, chamada de Problema do Brigadista com Vértices Resistentes, que se distingue pela presença de uma resistência ao fogo inteira associada a cada vértice. Apresentamos cinco heurísticas para o problema e os resultados obtidos em testes computacionais.

\section{Introdução}

O Problema do Brigadista, ou Firefighter Problem (FFP), foi introduzido pela primeira vez por Hartnell (1995). Neste problema tem-se um grafo não dirigido $G=(V, E)$, onde $V$ é o conjunto de vértices e $E$ é o conjunto de arestas, um conjunto $Q_{1}$ de vértices inicialmente queimados (focos de incêndio), e um inteiro $D$ representando o número de brigadistas, conforme Garcia-Martínez et al. (2015). Cada vértice de G pode encontrar-se nos estados queimado, defendido ou intocado. Em cada iteração, $D$ brigadistas estão disponíveis e cada um deles deve defender um único vértice que esteja no estado intocado. Em seguida, o fogo espalha-se para todos os vértices intocados que sejam adjacentes a algum vértice queimado. Um vértice queimado ou defendido permanecerá nesse estado nas iterações seguintes até o fim do processo, que ocorre quando o fogo não puder mais se espalhar, e portanto estará contido.

Neste artigo apresentamos uma nova variante do Problema do Brigadista, que chamaremos de Problema do Brigadista com Vértices Resistentes (FFPR). Nesta variante cada vértice $v \in \mathrm{V}$ possui inicialmente uma resistência $\lambda_{v}$. A cada iteração, a resistência de cada vértice intocado $v$ adjacente a algum vértice queimado é decrementada do número de vértices queimados adjacentes a $v$. No FFPR um vértice intocado $v$ se torna queimado quando $\lambda_{v}$ se torna menor ou igual a 0 . Desta forma, o FFPR é capaz de modelar cenários onde vértices não se incendeiam imediatamente após o contato com vizinhos queimados, considerando a resistência dos materiais sob exposição ao fogo. Essa variante também pode ser utilizada para modelar cenários na área de epidemiologia, onde os vértices representariam regiões, a resistência seria uma estimativa de contaminação da área e o fogo uma doença sem cura, de maneira semelhante ao apontado por Hartke et al. (2007). Vale ressaltar que uma instância do FFP pode ser considerada como uma instância do FFPR na qual a resistência inicial de todos os vértices é igual a 1. Na próxima seção descrevemos as heurísticas utilizadas para a resolução do FFPR e na última seção apresentamos os resultados obtidos a partir de simulações e tecemos algumas considerações finais. 


\section{Heurísticas para o FFPR}

Antes de apresentarmos as heurísticas propostas para o FFPR, precisamos de algumas definições. Seja $B$ um conjunto de vértices. Chamamos de vizinhança intocada de $B$, denotada por $N_{1}(B)$, o conjunto de todos os vértices intocados adjacentes a algum dos vértices de $B$. A k-vizinhança intocada de $B$, denotada por $N_{k}(B)$, é o conjunto de todos os vértices intocados adjacentes a algum dos vértices de $N_{k-1}(B)$. O grau dinâmico de um vértice $v$, é a quantidade de vértices intocados adjacentes a $v$. Observe que ao longo do processo os vértices intocados podem passar para o estado queimado ou defendido. Por esse motivo, o grau dinâmico dos vértices é alterado ao longo das iterações. Vamos denotar por $Q_{t}$ o conjunto dos vértices queimados no início da iteração $t$.

Para resolver o FFPR utilizamos uma heurística que chamamos de $k$-DynDegree, baseada na heurística DynDegree proposta em Ramos (2018). A heurística $k$-DynDegree consiste em, a cada iteração $t$, ordenar os vértices de $N_{k}\left(Q_{t}\right)$ (sendo $k$ um parâmetro a ser escolhido pelo usuário) primariamente em ordem não crescente de grau dinâmico e secundariamente em ordem não crescente de distância para os vértices queimados e então defender os $D$ primeiros vértices nessa ordem. Dessa maneira serão defendidos os vértices com maior grau dinâmico que estão mais distantes dos vértices queimados.

Usamos também uma nova heurística que denominamos de $k-M i n \lambda$. Tal heurística consiste em, a cada iteração $t$, ordenar os vértices de $N_{k}\left(Q_{t}\right)$ primariamente em ordem crescente de resistência e secundariamente em ordem não crescente de distância para os vértices queimados e então defender os $D$ primeiros vértices nessa ordem. Utilizamos também uma terceira heurística que chamamos de $k$-Random, que consiste em selecionar aleatoriamente $D$ vértices do conjunto $N_{k}\left(Q_{t}\right)$ para serem defendidos.

Experimentamos ainda combinar as heurísticas $k$-Min $\lambda$ e $k$-DynDegree para determinar os vértices a serem defendidos. Denominamos de $k$-Min $\lambda+k$-DynDegree a heurística na qual ordenamos os vértices de $N_{k}\left(Q_{t}\right)$ primariamente em ordem crescente de resistência, secundariamente em ordem não crescente de grau dinâmico e terciariamente em ordem não crescente de distância para os vértices queimados e então defendemos os $D$ primeiros vértices nessa ordem. A heurística busca defender os vértices com menor resistência, em caso de empate, defender os que têm maior grau dinâmico, e persistindo o empate defender os vértices mais distantes dos vértices queimados. Por fim, denominamos de $k$-DynDegree $+k$-Min $\lambda$ a heurística na qual ordenamos os vértices de $N_{k}\left(Q_{t}\right)$ primariamente em ordem não crescente de grau dinâmico, secundariamente em ordem crescente de resistência e terciariamente em ordem não crescente de distância para os vértices queimados e então defendemos os $D$ primeiros vértices nessa ordem.

\section{Resultados Numéricos}

Para a obtenção dos resultados apresentados nas tabelas 1 e 2, foi utilizada para a geração de instâncias de testes a biblioteca networkx disponível para Python 3. Em todas as instâncias havia inicialmente um único vértice queimado, determinado pseudoaleatoriamente. Os valores de $n$ das instâncias da tabela 1 foram obtidos das instâncias de teste de Garcia-Martínez et al. (2015), valendo-se, no entanto, de grafos obtidos a partir do modelo de Erdős-Rényi, com $n$ sendo o número de vértices no grafo e $p$ a probabilidade de existir uma aresta ligando quaisquer vértices distintos. Foram geradas 450 instâncias de grafos divididas em 9 classes diferentes (50 instâncias para cada classe), com cada 
classe sendo obtida a partir de uma combinação diferente de $n$ e $p$. Em toda classe, o valor médio de $\lambda_{v}$ foi de aproximadamente 3 .

Ademais, para cada classe de grafos foram obtidos resultados com $D=1$ e $D=2$ e foram usadas as cinco heurísticas apresentadas na seção anterior. Além disso, foram utilizados valores de $1 \leq k \leq 3$, e cada resistência $\lambda_{v}$ foi gerada pseudoaleatoriamente com um valor entre 1 e 5 . Na tabela $1, \overline{\operatorname{deg}(v)}$ representa a média dos graus dos vértices da classe, $\bar{t}$ é a média do número de iterações e $Q$ é a porcentagem média de vértices queimados ao fim da resolução das instâncias A linha Média indica a média obtida, seja de $\bar{t}$ ou de $Q$ para os valores daquela coluna. Cada valor em negrito indica o melhor resultado para aquela classe de instâncias.

Tabela 1. Resultados para modelo Erdős-Rényi (Heurísticas combinadas)

\begin{tabular}{|c|c|c|c|c|c|c|c|c|c|c|c|c|c|c|c|c|}
\hline \multirow{3}{*}{ classe } & \multirow[b]{3}{*}{$\mathrm{D}$} & \multirow[b]{3}{*}{$\mathrm{n}$} & \multirow[b]{3}{*}{$\mathrm{p}$} & \multirow[b]{3}{*}{$\overline{\operatorname{deg}(v)}$} & \multicolumn{6}{|c|}{ k-min $\lambda+$ k-dynDegree } & \multicolumn{6}{|c|}{ k-DynDegree + k-Min $\lambda$} \\
\hline & & & & & \multicolumn{2}{|c|}{$k=1$} & \multicolumn{2}{|c|}{$k=2$} & \multicolumn{2}{|c|}{$k=3$} & \multicolumn{2}{|c|}{$k=1$} & \multicolumn{2}{|c|}{$k=2$} & \multicolumn{2}{|c|}{$k=3$} \\
\hline & & & & & $\overline{\bar{t}}$ & $Q$ & $\overline{\bar{t}}$ & $Q$ & $\bar{t}$ & $Q$ & $\bar{t}$ & $Q$ & $\bar{t}$ & $Q$ & $\bar{t}$ & $Q$ \\
\hline & 1 & 50 & 0.06 & 3.09 & 3.5 & $3 \%$ & 6.8 & $11 \%$ & 9.1 & $19 \%$ & 4.2 & $8 \%$ & 8.8 & $24 \%$ & 10.6 & $31 \%$ \\
\hline 2 & 1 & 50 & 0.18 & 8.85 & 7.5 & $79 \%$ & 6.8 & $87 \%$ & 6.8 & $87 \%$ & 7.0 & $87 \%$ & 6.6 & $88 \%$ & 6.6 & $88 \%$ \\
\hline 3 & 1 & 50 & 0.54 & 26.32 & 4.1 & $94 \%$ & 4.0 & $94 \%$ & 4.0 & $94 \%$ & 4.0 & $94 \%$ & 4.0 & $94 \%$ & 4.0 & $94 \%$ \\
\hline 4 & 1 & 100 & 0.03 & 3.09 & 3.6 & $2 \%$ & 11.1 & $23 \%$ & 13.8 & $33 \%$ & 5.1 & $7 \%$ & 12.7 & $32 \%$ & 15.9 & $48 \%$ \\
\hline 5 & 1 & 100 & 0.09 & 8.88 & 8.7 & $80 \%$ & 8.2 & $92 \%$ & 8.2 & $92 \%$ & 8.4 & $90 \%$ & 7.8 & $93 \%$ & 7.9 & $93 \%$ \\
\hline 6 & 1 & 100 & 0.27 & 26.74 & 4.4 & $96 \%$ & 4.3 & $97 \%$ & 4.3 & $97 \%$ & 4.2 & $97 \%$ & 4.2 & $97 \%$ & 4.2 & $97 \%$ \\
\hline 7 & 1 & 500 & 0.006 & 3.08 & 5.0 & $6 \%$ & 13.6 & $34 \%$ & 21.3 & $65 \%$ & 6.3 & $11 \%$ & 21.2 & $64 \%$ & 23.9 & $77 \%$ \\
\hline 8 & 1 & 500 & 0.018 & 9.00 & 10.4 & $86 \%$ & 10.2 & $98 \%$ & 10.1 & $98 \%$ & 10.1 & $92 \%$ & 10.0 & $98 \%$ & 9.9 & $98 \%$ \\
\hline 9 & 1 & 500 & 0.054 & 26.95 & 5.6 & $99 \%$ & 5.4 & $99 \%$ & 5.4 & $99 \%$ & 5.4 & $99 \%$ & 5.3 & $99 \%$ & 5.3 & $99 \%$ \\
\hline 1 & 2 & 50 & 0.06 & 3.09 & 1.9 & $2 \%$ & 3.4 & $3 \%$ & 4.7 & $5 \%$ & 1.9 & $3 \%$ & 4.5 & $9 \%$ & 5.7 & $12 \%$ \\
\hline 2 & 2 & 50 & 0.18 & 8.85 & 6.6 & $36 \%$ & 7.3 & $67 \%$ & 7.6 & $70 \%$ & 6.7 & $59 \%$ & 6.8 & $75 \%$ & 6.7 & $75 \%$ \\
\hline 3 & 2 & 50 & 0.54 & 26.32 & 4.1 & $87 \%$ & 4.0 & $88 \%$ & 4.0 & $88 \%$ & 4.0 & $88 \%$ & 4.00 & $88 \%$ & 4.0 & $88 \%$ \\
\hline 4 & 2 & 100 & 0.03 & 3.09 & 1.9 & $1 \%$ & 4.3 & $3 \%$ & 7.1 & $8 \%$ & 2.1 & $2 \%$ & 5.8 & $8 \%$ & 9.3 & $17 \%$ \\
\hline 5 & 2 & 100 & 0.09 & 8.88 & 6.3 & $28 \%$ & 8.6 & $80 \%$ & 8.7 & $84 \%$ & 7.1 & $62 \%$ & 8.0 & $85 \%$ & 8.0 & $85 \%$ \\
\hline 6 & 2 & 100 & 0.27 & 26.74 & 4.6 & $93 \%$ & 4.3 & $93.2 \%$ & 4.3 & $93.2 \%$ & 4.3 & $93.2 \%$ & 4.2 & $93.4 \%$ & 4.2 & $93 \%$ \\
\hline 7 & 2 & 500 & 0.006 & 3.08 & 2.0 & $0.22 \%$ & 5.4 & $7 \%$ & 15.1 & $35 \%$ & 2.7 & $2 \%$ & 13.0 & $25 \%$ & 19.6 & $52 \%$ \\
\hline 8 & 2 & 500 & 0.018 & 9.00 & 7.9 & $44 \%$ & 10.1 & $94 \%$ & 10.1 & $96 \%$ & 9.4 & $81 \%$ & 9.8 & $94 \%$ & 9.8 & $96 \%$ \\
\hline \multirow[t]{2}{*}{9} & 2 & 500 & 0.054 & 26.95 & 5.8 & $98 \%$ & 5.4 & $98 \%$ & 5.4 & $98 \%$ & 5.4 & $98 \%$ & 5.3 & $98 \%$ & 5.3 & $98 \%$ \\
\hline & & & & Média & 5.2 & $52 \%$ & 6.8 & $65 \%$ & 8.3 & $70 \%$ & 5.5 & $60 \%$ & 7.9 & $70 \%$ & 8.9 & $75 \%$ \\
\hline
\end{tabular}

Tabela 2. Resultados para modelo Erdős-Rényi (Heurísticas individuais)

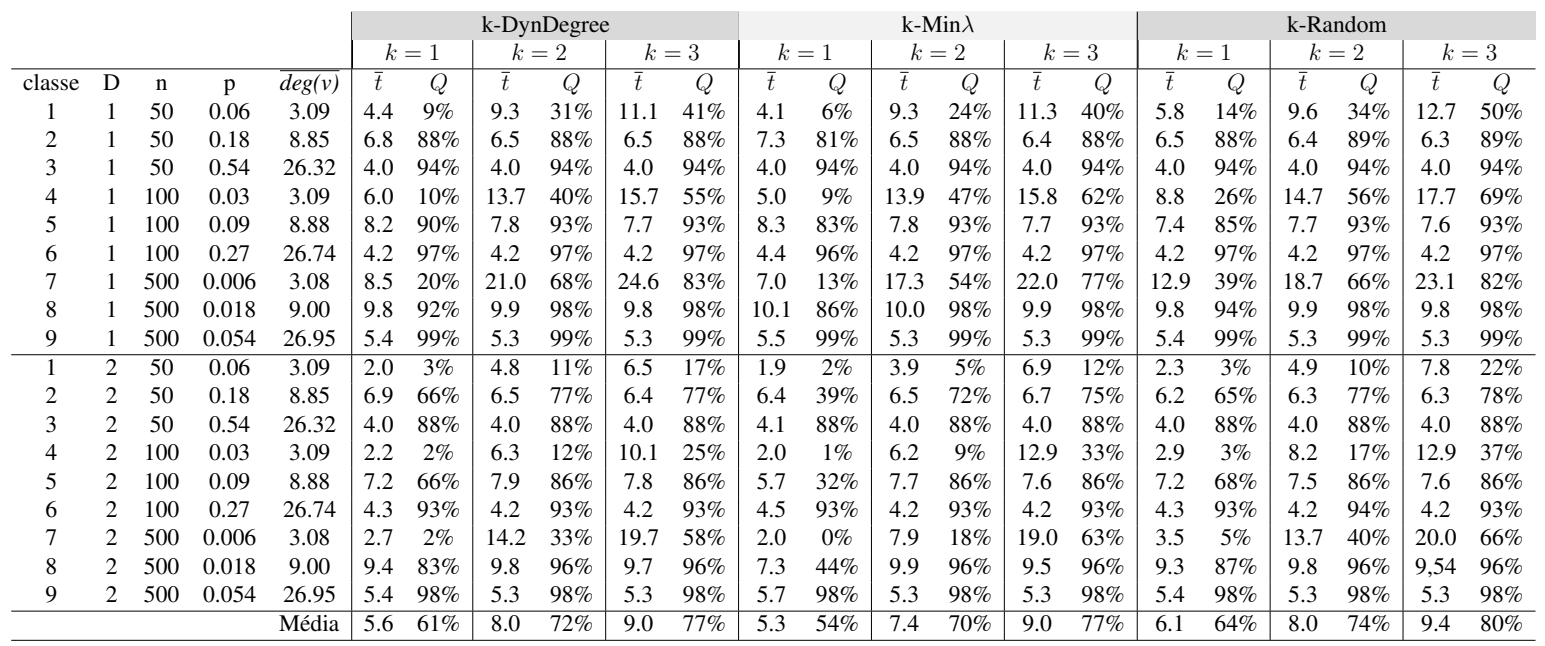

Uma análise das tabelas 1 e 2 nos permite observar que para todas as classes, seja $\operatorname{com} D=1$ ou $D=2$, o uso da heurística $k-\operatorname{Min} \lambda+k$-DynDegree resultou em uma média menor de vértices queimados quando comparado às demais heurísticas. Isso sugere que é preferível defender primeiramente os vértices que estão mais próximos de ser incendiados. Percebemos também que o valor de $k$ também influencia na quantidade de vértices 
queimados. Quanto maior o $k$, mais vértices queimados. Em nossos testes, defender vértices mais distantes do fogo não produziu bons resultados. Como esperado, o uso de $D=2$ resultou em menos vértices queimados em todas as classes de instâncias quando comparado com $D=1$. Percebemos ainda que o uso de $k$-Random resulta nos piores resultados, indicando que escolher aleatoriamente os vértices que serão defendidos não é uma boa estratégia. Para ambas as tabelas, aparenta haver uma relação entre $k$ e $\bar{t}$, com o número de iterações geralmente aumentando à medida que se aumenta $k$. Ainda, como era de se esperar, o uso de $D=2$ também resultou numa diminuição no número de iterações quando comparado com $D=1$. Por fim, podemos notar que nos grafos mais densos, independente da heurística utilizada, quase todos os vértices acabaram queimados.

Para a obtenção dos resultados da tabela 3, foram geradas 50 instâncias de grafos em grade de dimensões $25 \times 25$ (total de 625 vértices), com o foco localizado no centro do grafo, $D=1$ e $1 \leq k \leq 5$. Na linha de $\lambda$ aleatório, $\lambda_{v}$ foi gerado pseudoaleatoriamente entre 1 e 5 . Em cada tabela os valores representam a média de vértices que foram queimados para aquela combinação de $\lambda$ e $k$.

Tabela 3. Resultados com grafo em grade

\begin{tabular}{r|r|r|r|r|r|r|r|r|r|r|r|r} 
& \multicolumn{10}{|c}{$\mathrm{k}-\min \lambda+\mathrm{k}-$ dynDegree } \\
\multicolumn{1}{l|}{$\mathrm{k}^{\mathrm{k}} \mathrm{k}=1$} & $\mathrm{k}=2$ & $\mathrm{k}=3$ & $\mathrm{k}=4$ & $\mathrm{k}=5$ & $\mathrm{k}=6$ & $\mathrm{k}=1$ & $\mathrm{k}=2$ & $\mathrm{k}=3$ & $\mathrm{k}=4$ & $\mathrm{k}=5$ & $\mathrm{k}=6$ \\
\hline aleatório & $\mathbf{1 . 6 2}$ & 340.4 & 565.88 & 566.74 & 565.1 & 563.86 & 286.6 & 572.42 & 575.64 & 575.94 & 575.94 & 576.54 \\
\hline 1 & $\mathbf{5 9 9}$ & 601 & 601 & 601 & 600 & 599 & 601 & 600.88 & 600.96 & 600.84 & 600.82 & 600.7 \\
\hline 2 & $\mathbf{5 5 6}$ & 577 & 563 & 583 & 581 & 583 & 585.28 & 586.54 & 587.3 & 587.56 & 587.5 & 587.58 \\
\hline 3 & 2 & 6 & 543 & 561 & 554 & 563 & 2 & 528.2 & 570.08 & 570.94 & 571.92 & 571.84 \\
\hline 4 & 1 & 2 & 2 & 2 & 2 & 2 & 1 & 2 & 2 & 2 & 2 & 2 \\
\hline 5 & 1 & 1 & 1 & 1 & 1 & 1 & 1 & 1 & 1 & 1 & 1 & 1 \\
\hline
\end{tabular}

Percebemos que a seleção de vértices mais distantes dos vértices queimados, o que é mais frequente à medida que $k$ cresce, não se mostrou a escolha mais proveitosa. Mais uma vez observamos que a heurística $k-M i n \lambda+k$-DynDegree produz resultados melhores do que a heurística $k$-Random.

Pretendemos, em trabalhos futuros, fazer uso de técnicas como busca local e BRKGA para aprimorar resultados obtidos com nossas heurísticas. Pretendemos também formular o FFPR como um PLI e tentar resolvê-lo desta forma, de forma a possivelmente obter resultados ótimos que possam ser comparados com aqueles obtidos com o uso de heurísticas, de forma a melhor avaliar a eficácia de cada heurística utilizada.

\section{Referências}

Develin, M. and Hartke, S. G. (2007). Fire containment in grids of dimension three and higher. In Discrete Applied Mathematics, volume 155, pages 2257-2268. Elsevier.

García-Martínez, C., Blum, C., Rodriguez, F., and Lozano, M. (2015). The firefighter problem: Empirical results on random graphs. In Computers and Operational Research, pages 55-66. Elsevier.

Hartnell, B. (1995). Firefighter! an application of domination. Winnipeg, Canada. 25th Manitoba Conference on Combinatorial Mathematics and Computing.

Ramos, N. (2018). Um estudo computacional do problema do brigadista em grafos. Universidade Estadual de Campinas. 